\title{
ELASTIC MODULI OF A MEDIUM WITH LIQUID-FILLED CRACKS*
}

\author{
By H. D. GARBIN** ANd L. KNOPOFF (University of California, Los Angeles)
}

In earlier papers [1, 2], we have calculated the effective elastic modulus of a random medium permeated by a sparse distribution of circular cracks. The boundary conditions considered were those of vanishing normal stress on the surface of the cracks; this is usually called the free-free (f.f.) boundary condition.

The problem of earthquake prediction is of topical interest. A model which has been proposed to explain certain premonitory phenomena, involving elastic wave velocities through the focal zone of anticipated large earthquakes, involves wave propagation through a medium permeated by cracks which are air-filled and liquid-filled at different stages of the premonitory process [3]. The process of opening up cracks under large regional stress is called dilatancy in the seismic jargon. In the early stages of a premonitory episode, the cracks are partly air-filled; in the later stages, the cracks become fluid-filled.

The results obtained in the case of scattering from free-free cracks considered in [1] and [2] are pertinent to the determination of the properties of the dilatant material in the early stages of the premonitory episode. A consideration of the problem of the scattering of elastic waves by a medium permeated by liquid-filled (l.f.) cracks would complete the catalogue of properties needed to study this problem. In this note, we show that the solution to this problem can be obtained without significant additional calculation if we take advantage of the techniques and results already described in [1] and [2].

We consider, as before, the problem of the scattering of long wavelength elastic waves from an elastic medium in which a single circular crack of radius $a$ is imbedded. The crack has zero thickness, mathematically. Physically, the crack is filled with a nonviscous fluid. We use a cylindrical coordinate system fixed in the crack $(r, \phi, z)$. Then the boundary conditions for this problem are

$$
\begin{gathered}
\tau_{r z}+\tau_{r z}{ }^{0}=0 \\
\tau_{\phi z}+\tau_{\phi z}{ }^{0}=0 \\
\tau_{r z}, \tau_{z z}, \tau_{\phi z}, u_{r}, u_{\phi}, u_{z} \text { are continuous in the plane } z=0, r>a . \\
\tau_{z z} \text { and } u_{z} \text { are continuous for } z=0, r<a .
\end{gathered}
$$

In these expressions $\tau$ is the stress tensor for the scattered field, $u$ is the displacement vector of the scattered field and $\tau^{0}$ is the stress tensor for the incident field.

A comparison of the boundary conditions for the l.f. and f.f. cases shows that conditions (i), (ii) and (iii) are the same in both cases. However, condition (iv) for the f.f. case is

* Received May 29, 1974. Publication No. 1312, Institute of Geophysics and Planetary Physics, University of California, Los Angeles.

** Present address: Sandia Corporation, Albuquerque, N. M. 


$$
\tau_{z z}+\tau_{z z}{ }^{0}=0 ; \quad z \rightarrow 0 \pm, \quad 0 \leq r<a .
$$

Boundary conditions (i), (ii) and (iii) lead to coupled dual integral equations ([1], Eqs. $17 \mathrm{a}, \mathrm{b}$ and $8 \mathrm{a}, \mathrm{b}$ ). In the f.f. case, the condition (iv; f.f.) plus the condition (iii) of continuity of $u_{z}(r>a)$ lead to the integral

$$
\begin{gathered}
\int_{0}^{\infty} G(\zeta) P_{1 n}(\zeta) J_{n}(\zeta r) d \zeta=\left(2 \alpha^{2}-k_{2}{ }^{2}\right) i^{n} J_{n}(\alpha r), \quad 0 \leq r<1, \\
\int_{0}^{\infty} \frac{\nu_{1} P_{1 n}(\zeta) J_{n}(\zeta r)}{2 \zeta^{2}-k_{2}{ }^{2}} d \zeta=0, \quad r>1
\end{gathered}
$$

for the case of incident $P$-waves specified as in [1] (Eq. 14). Temporarily, the crack is assumed to have unit radius.

By means of the conditions of continuity of the stresses $\tau_{z i}$ across the plane $z=0$ for both the f.f. and l.f. cases, the jump in the normal component of displacement across the circular crack can be written as

$$
\begin{aligned}
{\left[u_{z}\right] } & =2{k_{2}}^{2} \sum_{n=-\infty}^{\infty} \exp (i n \phi) \int_{0}^{\infty} \frac{\nu_{1} P_{1 n}(\zeta) J_{n}(\zeta r) d \zeta}{2 \zeta^{2}-{k_{2}}^{2}}, & & r<1, \\
& =0, & & r>1,
\end{aligned}
$$

where the notation is the same as in [1] but we choose to recall especially that $\zeta^{-1} P_{1 n}(\zeta)$ is the part of the distribution function of the $n_{\mathrm{tb}}$ order scattered $P$-wave potential which corresponds to radiation symmetric in $z$ :

$$
\Phi=\sum_{n=-\infty}^{\infty} \exp (i n \phi) \int_{0}^{\infty}\left\{P_{1 n}(\zeta) \mp P_{2 n}(\zeta)\right\} J_{n}(\zeta r) \exp \left(-\nu_{1}|z|\right) d \zeta .
$$

In the l.f. case $\left[u_{z}\right]=0$, so we may write $P_{1 n}(\zeta)=0$. Hence the results for the l.f. case may be obtained directly from the f.f. case merely by setting $P_{1 n}(\zeta)=0$; this implies $s_{n}(\zeta)=0$. The results from [1] and [2] are

$$
\begin{gathered}
\frac{1}{\lambda+2 \mu}=\frac{1}{(\lambda+2 \mu)_{0}}\left\{1+\frac{64}{3 v} \sum_{p=1}^{N} a_{p}{ }^{3} \frac{\mu_{0}}{3 \lambda_{0}+4 \mu_{0}} \sin ^{2} \theta_{0 p} \cos ^{2} \theta_{0 p}\right\}, \\
\frac{1}{\mu}=\frac{1}{\mu_{0}}\left\{1+\frac{16}{3 v} \frac{\lambda_{0}+2 \mu_{0}}{3 \lambda_{0}+4 \mu_{0}} \sum_{p=1}^{N} a_{p}{ }^{3}\left[\cos ^{2} \theta_{0 p} \sin ^{2} \phi_{0 p}+\left(1-2 \cos ^{2} \theta_{0 p}\right)^{2} \cos ^{2} \phi_{0 p}\right]\right\},
\end{gathered}
$$

In the case of an isotropic distribution of liquid-filled cracks of equal radius, the moduli of the composite are

$$
\begin{aligned}
\left\langle\frac{1}{\lambda+2 \mu}\right\rangle & =\left(\frac{1}{\lambda+2 \mu}\right)_{0}\left\{1+\frac{128}{45} \frac{N a^{3}}{v} \frac{\mu_{0}}{3 \lambda_{0}+4 \mu_{0}}\right\}, \\
\left\langle\frac{1}{\mu}\right\rangle & =\left(\frac{1}{\mu}\right)_{0}\left\{1+\frac{32}{15} \frac{N a^{3}}{v} \frac{\lambda_{0}+2 \mu_{0}}{3 \lambda_{0}+4 \mu_{0}}\right\} .
\end{aligned}
$$

We note that the result for $\langle 1 /(\lambda+2 \mu)\rangle$ in the case of an isotropic distribution of cracks could have been obtained from the following physical argument. Filling the cracks with a fluid which transmits compressional stresses means that the bulk modulus $\langle K\rangle$ of the composite will be the same as that of the unflawed material $K_{0}{ }^{1}$. The statement $P_{1 n}=0$ fulfills this condition. Hence

1 The authors are indebted to B. Budiansky for pointing out this condition. 


$$
\langle\lambda+2 \mu\rangle-4 / 3\langle\mu\rangle=(\lambda+2 \mu)_{0}-4 / 3 \mu_{0} .
$$

If we substitute from (6) into this expression, the result (5) follows. However, we may comment that this physical argument would have been inadequate to give us the results (3), (4) for the anisotropic distribution of cracks.

\section{REFERENCES}

[1] H. D. Garbin and L. Knopoff, The compressional modulus of a material permeated by a random distribution of circular cracks, Quart. Appl. Math. 30, 453-464 (1973)

[2] H. D. Garbin and L. Knopoff, The shear modulus of a material permeated by a random distribution of free circular cracks, Quart. Appl. Math. (this number)

[3] C. H. Scholz, L. R. Sykes and Y. P. Aggarwal, Earthquake prediction: a physical basis, Science 181, 803-810 (1973) 